\title{
SF3B4 as an early-stage diagnostic marker and driver of hepatocellular carcinoma
}

\author{
Qingyu Shen ${ }^{1,2}$ \& Suk Woo Nam ${ }^{1,2, *}$ \\ ${ }^{1}$ Department of Pathology, College of Medicine, The Catholic University of Korea, Seoul 06591, Korea, ${ }^{2}$ Functional RNomics Research \\ Center, The Catholic University of Korea, Seoul 06591, Korea
}

An accurate diagnostic marker for detecting early-stage hepatocellular carcinoma (eHCC) is clinically important, since early detection of HCC remarkably improves patient survival. From the integrative analysis of the transcriptome and clinicopathologic data of human multi-stage HCC tissues, we were able to identify barrier-to-autointegration factor 1 (BANF1), procollagen-lysine, 2-oxoglutarate 5-dioxygenase 3 (PLOD3) and splicing factor $3 b$ subunit 4 (SF3B4) as early HCC biomarkers which could be detected in precancerous lesions of HCC, with superior capabilities to diagnose eHCC compared to the currently popular HCC diagnostic biomarkers: GPC3, GS, and HSP70. We then showed that SF3B4 knockdown caused G1/S cell cycle arrest by recovering p2 $7^{\text {kip1 }}$ and simultaneously suppressing cyclins, and CDKs in liver cancer cells. Notably, we demonstrated that aberrant SF3B4 overexpression altered the progress of splicing progress of the tumor suppressor gene, kruppel like factor 4 (KLF4), and resulted in non-functional skipped exon transcripts. This contributes to liver tumorigenesis via transcriptional inactivation of $p 27^{K i p 1}$ and simultaneous activation of Slug genes. Our results suggest that SF3B4 indicates early-stage HCC in precancerous lesions, and also functions as an early-stage driver in the development of liver cancer. [BMB Reports: Perspective 2018; 51(2): 57-58]

*Corresponding author. E-mail: swnam@catholic.ac.kr

https://doi.org/10.5483/BMBRep.2018.51.2.021

Received 17 January 2018

Keywords: Alternative splicing, Driver gene, Early-stage diagnostic marker, KLF4, SF3B4

Abbreviations: BANF1, barrier-to-autointegration factor 1; eHCC, early-stage hepatocellular carcinoma; GEO, gene expression omnibus; GPC3, glypican 3; GS, glutamine synthetase; HSP70, heat-shock protein 70; KLF4, Kruppel like factor 4; PLOD3, procollagen-lysine, 2-oxoglutarate 5-dioxygenase 3; SF3B4, splicing factor 3b subunit 4; TCGA, The Cancer Genome Atlas

Perspective to: Qingyu Shen, Jung Woo Eun, Kyungbun Lee et al (2017) Hepatology. Accepted online 2017. 10. 23. doi: 10.1002/ hep. 29606
Hepatocellular carcinoma (HCC), the most common primary malignancy of the liver, is the sixth most common malignancy and the second leading cause of cancer-related mortality in the world (Torre LA et al (2012) CA Cancer J Clin 65, 87-108). The most curative treatment for HCC patients is surgical resection at an early stage, but very few patients are diagnosed at an early stage. Meanwhile, the cure rates for chemotherapies such as sorafenib and the receptor tyrosine kinase inhibitors (unitinib, brivanib, linifanib), are limited and show no advantages for overall survival and disease-free survival rates. Currently, some molecular biomarkers, such as $\alpha$-fetoprotein (AFP), glypican 3 (GPC3), glutamine synthetase, (GS) and heat-shock protein 70 (HSP70), are available for HCC diagnosis in clinical practice, but the sensitivity and specificity are not satisfactory Therefore, the discovery of an objective molecular biomarker that would help to standardize histologic diagnosis of early-stage HCC and lead to appropriate treatment is eagerly anticipated.

Like other cancers, HCC is characterized by a multi-step process leading to tumor progression. Based on this premise, to identify early-stage HCC decision molecular features, we performed whole transcriptome assays with 107 human hepatic tissues, including normal liver $(\mathrm{NL})$, fibrosis of chronic hepatitis $(\mathrm{CH})$, cirrhosis (CS), low-grade dysplastic nodule (LGDN), high-grade dysplastic nodule (HGDN) and HCCs, comprising histologic gradings of Edmonson Grade 1 (G1), G2 and G3. Hierarchical clustering analysis indicated that HGDN is a precancerous lesion connoting a driver gene signature, and progresses favorably to overt HCC. Based on these results, we designed a gene selection strategy to identify gene sets that would enable us to anticipate potential HCC within a precancerous lesion such as HGDN. Using various computational approaches, we selected 10 gene elements as candidates and, through immunohistochemical staining, showed that barrier-to-autointegration factor 1 (BANF1), procollagen-lysine,2-oxoglutarate 5-dioxygenase 3 (PLOD3) and splicing factor $3 \mathrm{~b}$ subunit 4 (SF3B4) are HCC diagnostic biomarkers, with superior capabilities to diagnose early-stage HCC in a large cohort of HCC patients, when compared to the currently popular trio of HCC diagnostic biomarkers i.e., GPC3, GS, and HSP70.

Alternative splicing (AS) is one of the main steps of the gene

ISSN: 1976-670X (electronic edition)

Copyright (C) 2018 by the The Korean Society for Biochemistry and Molecular Biology

(c) This is an open-access article distributed under the terms of the Creative Commons Attribution Non-Commercial License (http://creativecommons.org/licenses/by-nc/4.0) which permits unrestricted non-commercial use, distribution, and reproduction in any medium, provided the original work is properly cited. 


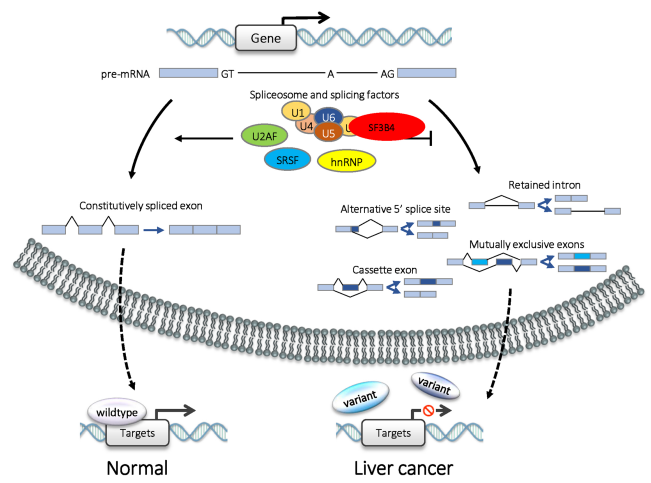

Diagram 1. Proposed schematic illustrates driver function of SF3B4 in early hepatocarcinogenesis. At the early-stage of HCC, SF3B4 overexpression triggers aberrant splicing of KLF4 to produce non-functional transcripts, which, in turn, suppresses the wild-type tumor suppressor gene, KLF4. Resultant suppression of KLF4 ${ }^{\text {WT }}$ contributes to activate malignant transformation and growth of hepatocytes via disturbance of the cell cycle and epithelialmesenchymal transition (EMT) regulation by suppressing $\mathrm{p} 27^{\mathrm{kip} 1}$ and simultaneously inducing Slug expression in HCC.

transcription process and contributes to proteome diversity. It is estimated that over $90 \%$ of genes have more than one transcript isoform generated through alternative splicing mechanism in humans. Several mRNA isoforms specific to certain cellular developmental states and diseases, including tumorigenesis, have been investigated. Also, several splicing factors were reported as oncogenes or tumor suppressor genes and regulate AS of genes in cancer progression. RNA splicing is regulated by two spliceosomes, the major (U2 snRNP dependent) and minor (U12 snRNP dependent) spliceosomes. U2 snRNP is composed of U2 snRNA and the splicing factor 3a, 3b (SF3a, SF3b) complex and form the major spliceosome. Among the six subunits of the SF3b complex, only SF3B4 was identified to be the most upregulated gene in HCC patients from the gene expression analyses of The Cancer Genome Atlas Liver Hepatocellular Carcinoma (TCGA_LIHC) data collection and the Gene Expression Omnibus (GËO) database of the National Center for Biotechnology Information (NCBI). In addition, SF3B4 was also frequently amplified (amplification: $11 \%$, gain: $60 \%$ ) in HCC patients and the expression and copy number values were highly correlated (Pearson $r=0.64$ ).

To investigate the biological function of SF3B4 in HCC, we performed RNAi-mediated gene knockdown followed by several cell phenotype assays. Target disruption of SF3B4 showed inhibition of HCC cell growth, proliferation, migration and invasion in vitro. SF3B4 knockdown induced G1 phase cell cycle arrest, not apoptosis, through upregulating of $\mathrm{p} 27^{\mathrm{Kip} 1}$ and down-regulating of cyclins and CDKs in HCC cells. To investigate whether SF3B4 upregulation could induce alternative splicing in $\mathrm{HCC}$, we performed AS analysis of SF3B4 knockdown- or Spliceostatin A (SSA, a SF3b complex inhibitor)-treated HCC cells. Both SF3B4 knockdown and
SSA-treated cells exhibited aberrant vascular endothelial growth factor (VECF) gene alternative splicing event and resulted in the suppression of wild-type VEGF and accordingly, upregulation of VEGF pre-mRNA in HCC cells. Next, to investigate the direct target of SF3B4 in hepatocarcinogenesis, we analyzed the NGS RNA-seq data of the SF3B4 knockdown HepG2 cells available from the GEO database (GSE80861), and we identified 8 gene elements that are upregulated in $\mathrm{HCC}$, with frequent AS events. After experimental validation in five different $\mathrm{HCC}$ cell lines, we found that kruppel-like factor 4 (KLF4) was directly regulated by SF3B4 in HCC cells. Direct sequencing analysis confirmed that the two non-functional alternative KLF4 transcripts were generated through skipped exon (SE) alternative splicing event. The dual luciferase reporter assay also demonstrated that KLF4 regulates $p 27^{\text {Kip } 1}$ and Slug expression by direct binding to their promoter regions. These results suggest that inactivation of KLF4 through aberrant expression of SF3B4 contributes to malignant transformation and growth of hepatocyte via transcriptional inactivation of $p 27^{K i p 1}$ and simultaneously activated Slug (Diagram 1).

Alternative splicing has been reported as an important mechanism in tumorigenesis in many cancers, and inhibitors for splicing factors were considered as new drugs for cancer therapy. Several natural products (like FR901464, Herboxidiene and Pladienolides) targeting the SF3b complex were reported to show anticancer effects, with low half maximal inhibitory concentrations $\left(\mathrm{IC}_{50}\right)$ and cytotoxic effects in animal models. EA. Obeng et al showed that Sf3b1 ${ }^{\mathrm{K} 700 \mathrm{E}}$ expression causes earlier onset of myelodysplastic syndrome (MDS) and develop macrocytic anemia and myelodysplasia in mice. Furthermore, cells expressing $S f 3 b 1^{K 700 E}$ showed more sensitivity $\left(\mathrm{IC}_{50}=\right.$ $0.619 \mathrm{nM})$ to $\mathrm{E} 7107$ than cells expressing wild-type Sf3b1 $\left(\mathrm{IC}_{50}=\right.$ $1.249 \mathrm{nM}$ ) in a mouse MDS model (EA. Obeng et al (2016) Cancer Cell 30, 404-417). In our study, we intravenously delivered the SF3B4-specific siRNA using nanoparticles to $\mathrm{H}$-ras transgenic mice, which spontaneously develop HCC. Our results showed that the mice injected with SF3B4-specific siRNA developed liver tumors much more tardily and smaller than that of the control-group (control siRNAs) mice. Overall, our findings suggest that novel molecular biomarkers, BANF1, PLOD3 and SF3B4 indicating early-stage HCC in a precancerous lesion, and that aberrantly over-expressed SF3B4 plays an important role as a cancer driver gene at early-stage of $\mathrm{HCC}$ and regulates the expression of many cancer-associated genes through an AS mechanism. AS is a considerable mechanism for cancer development and inhibition of AS may be a promising therapeutic strategy for HCC and many other cancers.

\section{ACKNOWLEDGEMENTS}

This work was supported by a grant from the National Research Foundation (NRF) of South Korea (2017R1A2B3002989). 